\title{
SEROLOGIC RESPONSES OF KOREAN SOLDIERS SERVING IN MALARIA-ENDEMIC AREAS DURING A RECENT OUTBREAK OF PLASMODIUM VIVAX
}

CHAE GYU PARK, YONG-JOON CHWAE, JONG-IL KIM, JI-HO LEE, GANG MIN HUR, BYEONG HWA JEON, JAE SOO KOH, JAE-HEE HAN, SHIN-JE LEE, JAE-WON PARK, DAVID C. KASLOW, DANIEL STRICKMAN, AND CHEON-SEOP ROH

Korean Armed Forces Central Medical Research Institute, Chumok-dong, Yuseong-gu, Taejeon, Republic of Korea; Laboratory of Malaria Research, National Institute of Allergy and Infectious Diseases, National Institutes of Health, Bethesda, Maryland; Fifth Medical Detachment, Eighteenth Medical Command, United States Forces Korea, Yongsan Garrison, Seoul, Republic of Korea

Abstract. Anti-Pv200 antibody levels were assessed in samples from endemic areas of Plasmodium vivax malaria in the Republic of Korea (ROK), using an indirect enzyme-linked immunosorbent assay (ELISA) method. Asymptomatic carriers of $P$. vivax were detected using nested polymerase chain reaction (PCR) of blood samples. AntiPv200 antibody levels in 20 vivax malaria patients (optical density \pm standard deviation [OD \pm SD] values $1.85 \pm$ 0.29 of IgG isotype and $1.33 \pm 1.33$ of IgM isotype) were markedly higher than those of uninfected, malaria-naive controls $(0.08 \pm 0.16$ of $\mathrm{IgG}$ isotype and $0.04 \pm 0.04$ of IgM isotype). Antibody levels for 7 out of 8 soldiers with a recent malaria infection were sustained above the cut-off values for 4 months after successful treatment. Analysis of serum collected from 40 healthy, asymptomatic soldiers who had a $P$. vivax malaria attack within 3 months after our sampling, revealed 11 antibody-positive samples (27.5\%), compared to 5 positive samples (12.5\%) collected from a random selection of 40 soldiers. Among a larger pool of 1,713 soldiers who had served in high-risk areas for $P$. vivax transmission, $15 \%$ were antibody positive. Among 1,000 blood samples from asymptomatic soldiers who had served in the high-risk areas, 4 samples $(0.4 \%)$ were parasite positive, as determined by nested PCR. Our results show that anti-Pv200 antibody levels can provide useful information in the late diagnosis of $P$. vivax malaria infection in a previously naive population and also in large seroepidemiologic studies. Furthermore, our results suggest that asymptomatic $P$. vivax carriers could be important in the current outbreak of malaria in Korea.

\section{INTRODUCTION}

Although previously known as an endemic area of Plasmodium vivax, the Republic of Korea (ROK) had been considered free from malaria parasites because no reports of autochthonous transmission had been documented since 1984. However, since re-emergence of a single case of $P$. vivax malaria in 1993, the number of malaria cases has increased exponentially in the northwestern part of the ROK (northern Kyunggi Province), causing more than 1,700 cases in 1997, and an estimated 4,000 cases in $1998 .^{1-4}$ Historically, Korean $P$. vivax malaria has an atypically long incubation period (lasting up to a year or longer) in a large proportion of patients but a typically mild clinical course ${ }^{5}$ characteristics which appear to apply to the current epidemic. Serologic responses to malaria parasite infections in Korea have not been reported previously, although serology would be a logical approach to detect infection during the long incubation period. The indirect enzyme-linked immunosorbent assay (ELISA) has been developed to detect and monitor antibodies against cell extracts, circumsporozoite (CS) antigen, or merozoite surface protein 1 (MSP 1) of Plasmodium spp. ${ }^{6-10}$ The indirect ELISA method could be a simple, inexpensive approach for diagnosis of asymptomatic carriers, as well as for the seroepidemiologic study of a large number of populations.

In this study, we measured antibody levels against Pv200 antigen, the MSP 1 homolog in P. vivax (Sal I strain), and against $P$. vivax CS protein by indirect ELISA of serum from infected and uninfected ROK Army soldiers who had served in high risk areas of $P$. vivax transmission. We also searched for the presence of circulating $P$. vivax parasites by performing nested PCR on subjects' blood. In this report, we demonstrate that the anti-Pv200 antibody levels might provide useful information in diagnosis and prognosis of the popu- lation exposed to Korean $P$. vivax malaria, as well as in large seroepidemiologic studies. We were also able to detect asymptomatic $P$. vivax carriers by nested PCR.

\section{MATERIALS AND METHODS}

Subjects and sample collection. This study conducted by the Korean Armed Forces Central Medical Research Institute was approved and reviewed annually by the Research Review Board in the ROK Armed Forces Medical Command. All participating soldiers were informed of the study objectives and on-going threats of Korean $P$. vivax malaria; subjects provided written personal histories and signed informed consent for their participation prior to sampling.

In late May 1997, samples for serum and whole blood were collected by venipuncture from 1,000 randomly selected Korean soldiers who had served in areas of northern Kyunggi Province, Korea, with high risk of $P$. vivax transmission during the summer of 1996 . These soldiers were still stationed in the $P$. vivax malaria-endemic areas at the time of sampling in May 1997, but sometime after the autumn of 1996 they had left these high-risk malarious areas to other service locations where the risk of $P$. vivax transmission was much lower. According to the report by the ROK Armed Forces Medical Command, 40 of the 1,000 soldiers sampled in May 1997, were later diagnosed and treated for $P$. vivax malaria. The emergence of these cases was within 3 months after our sampling.

For the second round of our large seroepidemiological survey, we collected serum from 1,713 Korean soldiers, beginning in early January 1998, who had served in high-risk areas of northern Kyunggi Province since the summer of 1997. Of these, 8 soldiers who had a previous history of $P$. vivax malaria could be sampled twice more during the subsequent 5 months. 
Negative control sera from malaria-naive subjects were collected from 113 soldiers in the Korean Armed Forces Medical School and from 200 civilians (kindly provided by the Korean Red Cross). None of the 313 subjects who donated negative control sera had a previous history of malaria or temporary residence in malaria-endemic areas.

Positive control sera were obtained from 20 malaria patients who had been admitted to ROK military field hospitals of northern Kyunggi Province and had been confirmed by examination of peripheral blood film smears during the summers of 1997 and 1998.

Indirect ELISA. The $19 \mathrm{kDa}$ fragment of Pv200, merozoite surface protein 1 (MSP 1) of $P$. vivax (Sal I strain), was expressed in the yeast Saccharomyces cerevisiae. The expression, purification, and characterization of this antigen have been described in detail elsewhere. ${ }^{9}$ V20, CS capture antigen containing 20 tandem repeats of predominant, also known as Pv210, nonapeptide (GDRAA/DGQPA) from $P$. vivax CS protein, was kindly provided by Dr. Robert A. Wirtz (Centers for Disease Control and Prevention). ${ }^{10}$ Titers of antibodies against Pv200 and V20 were determined by an indirect ELISA procedure with minor modifications from Kaslow and others. ${ }^{9}$ and Wirtz and others. ${ }^{10}$ Briefly, 96-well microtiter plates (Costar, Corning, NY) were coated with $0.1 \mu \mathrm{g}$ of antigen in phosphate buffered saline (PBS) at $4^{\circ} \mathrm{C}$ overnight. The plates were washed with PBS containing $0.05 \%$ Tween20 (Sigma, St. Louis, MO) (PBST), blocked with $5 \%$ goat serum (Sigma) in PBST at $37^{\circ} \mathrm{C}$ for $1 \mathrm{hr}$, and then washed with PBST to remove unbound proteins. Test sera diluted 1:100 were added to the wells and incubated at $37^{\circ} \mathrm{C}$ for $1 \mathrm{hr}$. Then the plates were washed with PBST and incubated with horseradish peroxidase-conjugated, anti-human IgG or IgM antibodies (Sigma) for another $1 \mathrm{hr}$. The bound antibodies were detected following incubation with $100 \mu \mathrm{l}$ of O-phenylene diamine (OPD) substrate (Sigma) using a microplate reader at $490 \mathrm{~nm}$ wavelength. The results were reported in units of optical density (OD) \pm standard deviation (SD). Every anti-Pv200 ELISA experiment included at least 3 negative naive individuals and 3 positive patient samples. Each serum sample was tested at least 3 times, except in the case of the high numbered seroepidemiologic survey where only positive samples were reconfirmed. Virtually identical ELISA results were obtained from each sample tested throughout the study.

DNA template preparation and nested PCR. To detect blood stage parasites of $P$. vivax, nested PCR amplification was applied according to the method of Snounou and others. ${ }^{11}$ To prepare the $P$. vivax DNA template, frozen whole blood samples containing EDTA were thawed at room temperature and diluted in 10 volumes of PBS with $0.05 \%$ saponin (Sigma). The parasites were released from erythrocytes at room temperature for $30 \mathrm{~min}$ and collected by centrifugation $(6000 \times \mathrm{g}$ for $5 \mathrm{~min})$. The supernatant was discarded; the parasite and leukocyte pellet was resuspended in $25 \mu \mathrm{l}$ of PCR buffer without $\mathrm{MgCl}_{2}$; and the mixture was incubated at $95^{\circ} \mathrm{C}$ for $10 \mathrm{~min}$. The first round of nested PCR amplification was then performed with a $5 \mu \mathrm{l}$ aliquot of supernatant from this mixture solution. For the first round of PCR amplification, two Plasmodium genus-specific primers, rPLU6 (5'-TTA AAA TTG TTG CAG TTA AAA CG-3') and rPLU5 (5'-CCT GTT GTT GCC TTA AAC TTC-3')

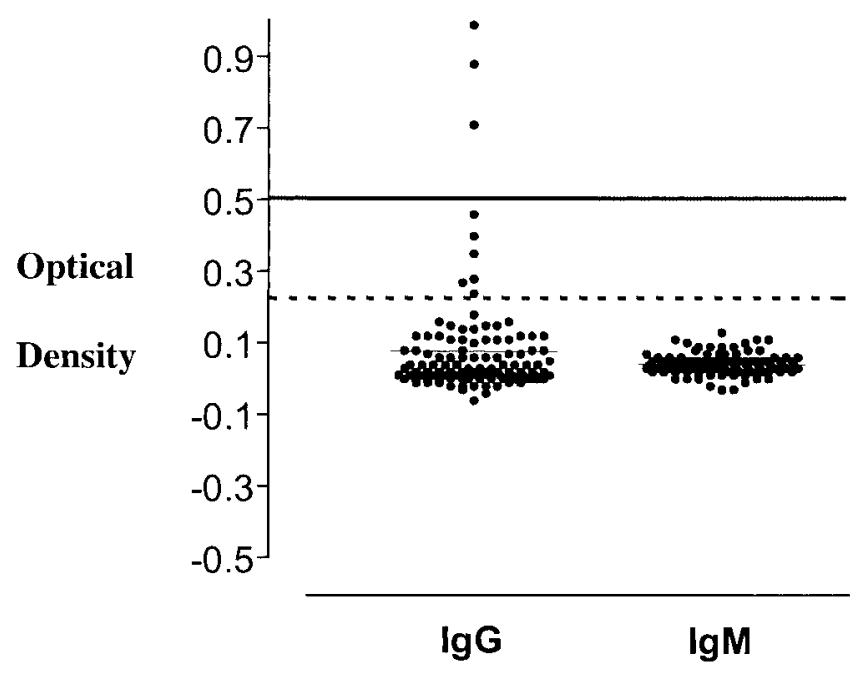

FIGURE 1. Anti-Pv200 antibody levels in a normal control group determined by indirect enzyme-linked immunosorbent assay (ELISA). The thick solid horizontal line and dotted horizontal line represent the cut-off values of optical densities (OD) in anti-Pv200 IgG isotype (0.5) and IgM isotype (0.2) antibodies, respectively. The thin horizontal lines indicate the mean OD in each anti-Pv200 antibody titer.

were used for amplification of small subunit ribosomal RNA (ssrRNA) genes. The second round of amplification was performed with a $1 \mu l$ aliquot of product from the first round of PCR, using species-specific primers of rVIV1 (5'-CGC TTC TAG CTT AAT CCA CAT AAC TGA TAC-3') and rVIV2 (5'-ACT TCC AAG CCG AAG CAA AGA AAG TCC TTA- $\left.3^{\prime}\right)$ to produce a $P$. vivax-specific $120 \mathrm{bp}$ fragment of ssrRNA genes. All PCR reactions were performed in 2 $\mathrm{mM} \mathrm{MgCl}{ }_{2}, 50 \mathrm{mM} \mathrm{KCl}, 10 \mathrm{mM}$ TrisCl (pH 8.3), $0.1 \mathrm{mg} /$ $\mathrm{ml}$ gelatin, $125 \mu \mathrm{M}$ of each of the four dNTPs, $250 \mathrm{nM}$ of each oligonucleotide primer, and 0.4 unit of AmpliTaq polymerase (Perkin Elmer Cetus).

\section{RESULTS}

Antibody levels in control group and malaria patients. To determine the cut-off values of anti-Pv200 antibody levels and to evaluate the usefulness of the indirect ELISA method for anti-Pv200 antigen in diagnosing $P$. vivax malaria in Korea, anti-Pv200 antibody levels were measured in sera of 313 normal individuals who had never been exposed to malaria parasites and 20 patients ill with active malaria. As shown in Figure 1, mean anti-Pv200 levels of $\mathrm{IgG}$ and IgM isotype antibodies in normal control sera were OD \pm SD of $0.08 \pm 0.16$ and $0.04 \pm 0.04$, respectively. Based on these results, OD values of 0.5 and 0.2 (mean $+3 \times \mathrm{SD}$ ) were chosen as the lower limits of positive reactions for IgG and IgM ELISA results, respectively (these cut-off values exclude $>97 \%$ of the control group). Anti-Pv200 antibody levels of 20 Korean $P$. vivax malaria patients had mean values of $1.85 \pm 0.29$ for the $\operatorname{IgG}$ isotype and $1.33 \pm 0.29$ for the IgM isotype (Figure 2). All of the patients' sera were above the cut-off values, except for one case that was below the cut-off for IgM.

Changes in antibody levels with a history of malaria. Serum samples from 8 individual soldiers with recent illness 


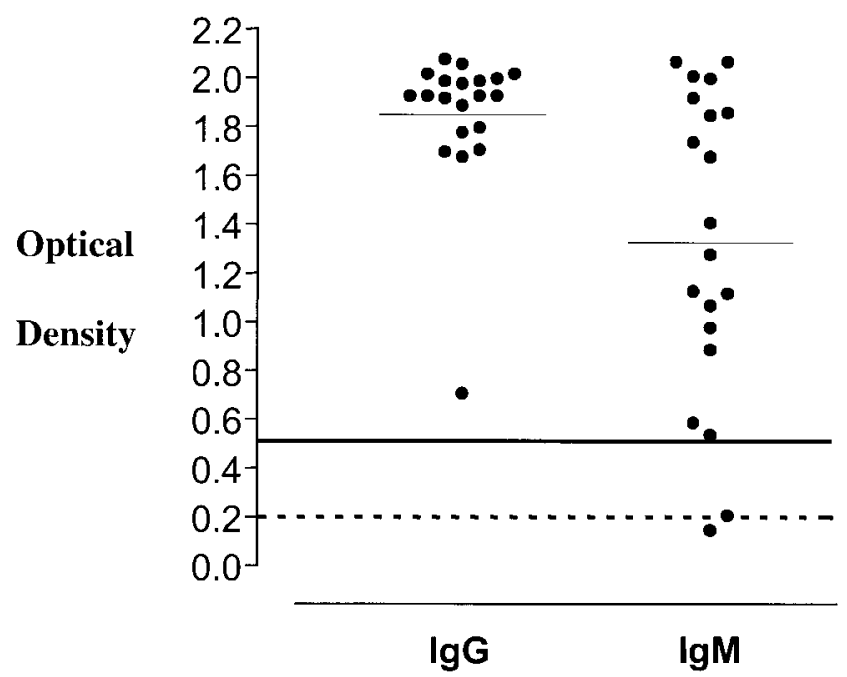

Figure 2. Anti-Pv200 antibody levels in Plasmodium vivax malaria patients determined by indirect enzyme-linked immunosorbent assay (ELISA). The thick solid horizontal line and dotted horizontal line represent the cut-off values of optical densities (OD) in IgG isotype (0.5) and $\operatorname{IgM}$ isotype (0.2) antibodies, respectively. The thin horizontal lines indicate the mean values of OD in each anti-Pv200 antibody titer.

from $P$. vivax infection were collected at 4,6 , and 9 months after recovery following treatment with chloroquine/primaquine. The anti-Pv200 IgG antibody levels were sustained above the limit of positive values for 4 months in all but one of the soldiers tested, and for 6 months in 3 of the soldiers tested (Figure 3).

Elevated antibody levels before malaria attack. According to the report by the ROK Armed Forces Medical Command, 40 of the 1,000 soldiers sampled in May 1997, had an attack of $P$. vivax malaria within 3 months after our sampling. Since these soldiers left the high-risk malarious area after autumn 1996, we assumed that their illnesses were
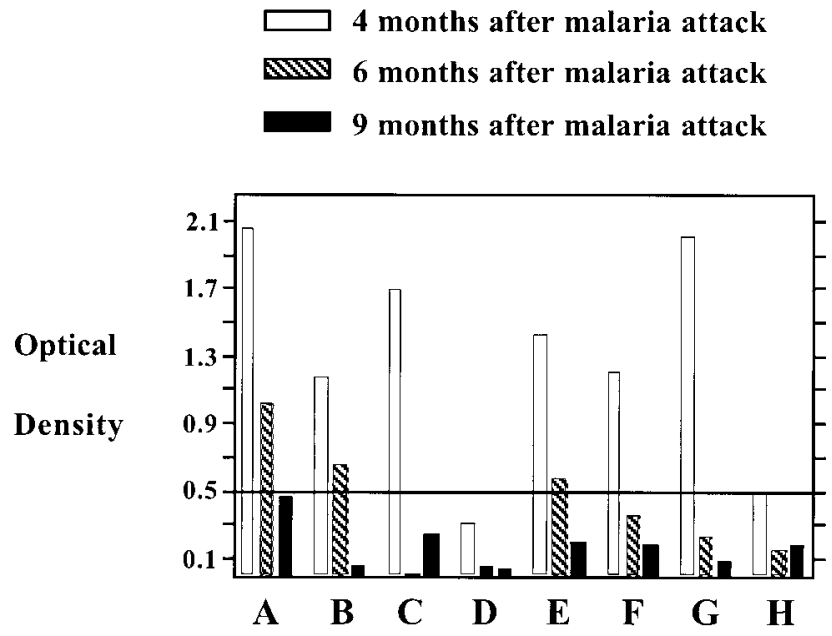

Figure 3. Anti-Pv200 antibody levels of 8 individual soldiers (designated A through $\mathrm{H}$ ) with a history of Plasmodium vivax malaria determined by indirect enzyme-linked immunosorbent assay (ELISA). Anti-Pv200 antibody titers of the serum samples from these 8 soldiers were tested at 4,6 , and 9 months after the malaria attack.
A

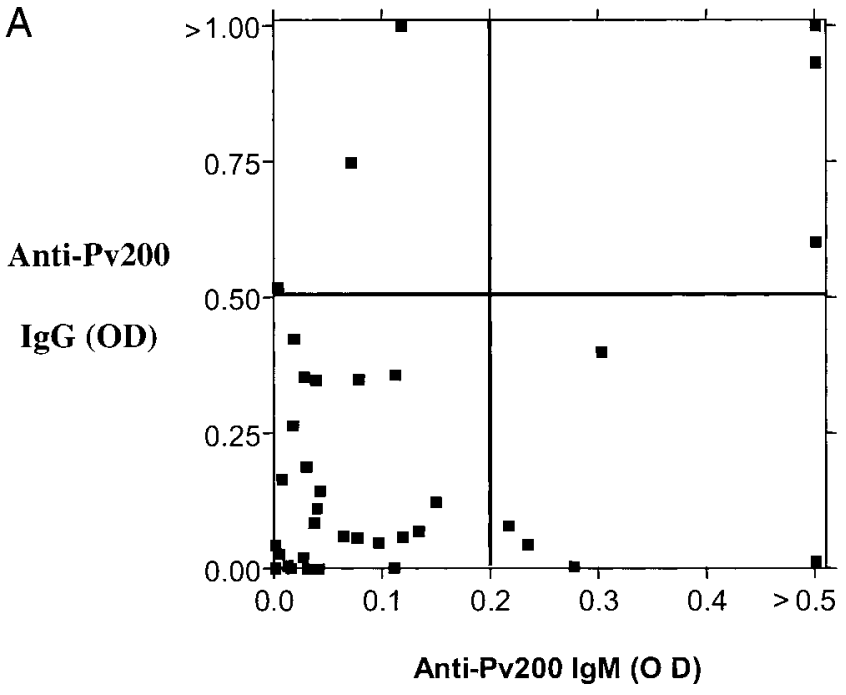

B

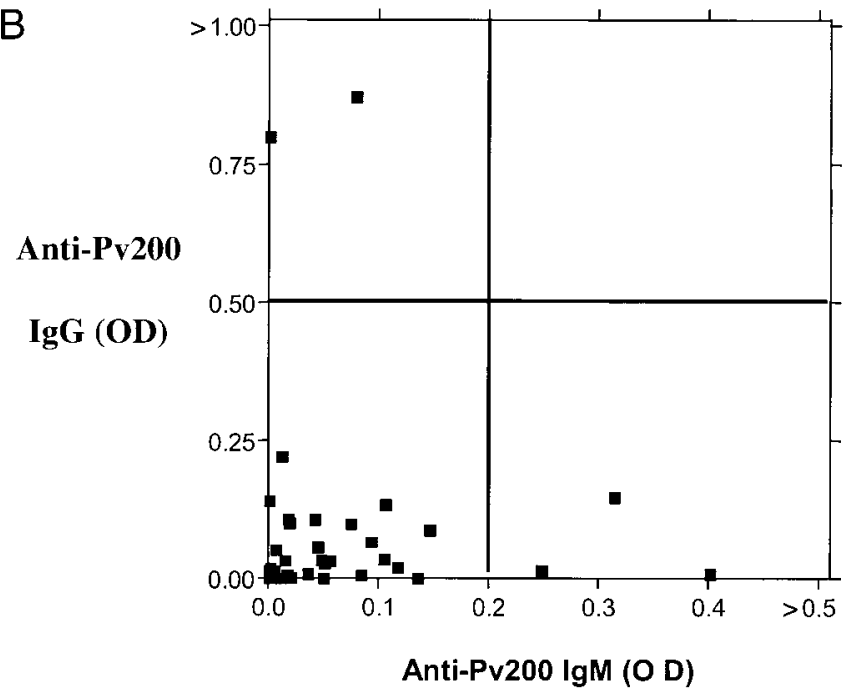

Figure 4. A): Anti-Pv200 antibody levels of serums from 40 soldiers who had served in high-risk Plasmodium vivax malaria areas and had malaria within 3 months following our sampling. The horizontal and vertical lines represent the cut-off values of optical densities (OD) in anti-Pv200 IgG isotype (0.5) and IgM isotype (0.2) antibodies, respectively. B): Anti-Pv200 antibody levels of serum from 40 randomly selected soldiers in our sampling pool who had served in high-risk Plasmodium vivax malaria areas. The horizontal and vertical lines represent the cut-off values of optical densities in anti-Pv200 IgG isotype (0.5) and IgM isotype (0.2) antibodies, respectively.

caused by infection acquired during the summer of 1996 . Anti-Pv200 antibody levels of these 40 soldiers were compared to the levels of 40 randomly selected soldiers from the pool of 1,000 (Figure 4). Eleven (27.5\%) of the serum samples were positive for anti-Pv200 IgG and IgM in the 40 soldiers who subsequently experienced a malaria attack, while only five $(12.5 \%)$ sera had positive values among the 40 randomly selected soldiers. The value distribution of antiPv200 antibody levels for the remaining 920 soldiers was virtually identical (12.7\% positive for anti-Pv200 IgG and $\mathrm{IgM}$, data not shown) to that of the 40 randomly selected soldiers.

Antibody levels in high risk areas. In late January 1998, 


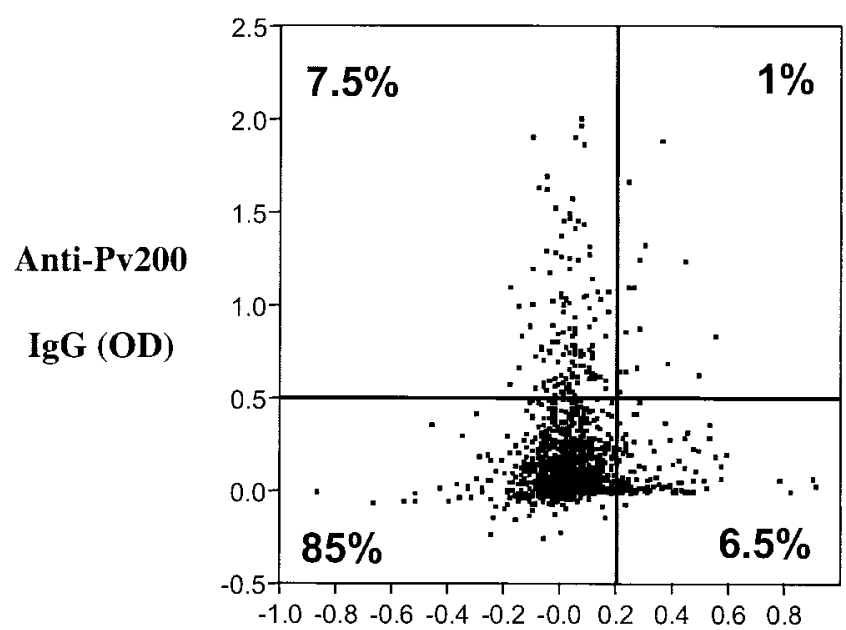

Anti-Pv200 IgM (O D)

FIGURE 5. Anti-Pv200 antibody levels of 1,713 soldiers who had been serving in high-risk Plasmodium vivax malaria areas, as determined by indirect enzyme-linked immunosorbent assay (ELISA). The horizontal and vertical lines represent the cut-off values of optical densities in anti-Pv200 IgG isotype (0.5) and IgM isotype $(0.2)$ antibodies, respectively.

we collected 1,713 serum samples from soldiers who had been serving since the summer of 1997 in areas with high malaria transmission rates. Of all the samples tested, $15 \%$ were positive for anti-Pv200 IgG or IgM (Figure 5).

Antibody levels against $P$. vivax CS protein. Antibody levels against $P$. vivax CS protein were tested with V20 antigen in four groups shown in Figure 6: A) 20 malaria patients; B) 12 soldiers within 6 months after malaria attack; C) 40 soldiers who had malaria within 3 months after sampling; and D) 40 soldiers who did not have a malaria attack within 3 months after sampling. The mean IgG antibody titer of group A patients was $0.40 \pm 0.76$, which was not significantly higher than those for the other groups (B, $0.15 \pm$ 0.37 ; C, $0.24 \pm 0.09$; and D, $0.06 \pm 0.09$ ) (Figure 6A). Likewise, the mean IgM antibody level for group A was not significantly higher $(0.37 \pm 0.53)$ compared to those for the other groups $(\mathrm{B}, 0.03 \pm 0.53$; , OD $0.01 \pm 0.02$; and $\mathrm{D}$, $0.02 \pm 0.02$ ) (Figure 6B).

Detection of $\boldsymbol{P}$. vivax in blood. Among 1,000 whole blood samples collected in late May 1997, $P$. vivax parasites were detected in 4 soldiers by nested PCR (Figure 7). These soldiers had no clinical signs or symptoms of malaria at the time of our sampling.

\section{DISCUSSION}

The present serologic study clearly demonstrated that distinct anti-Pv200 antibody responses occur in the course of $P$. vivax infection experienced during the recent malaria epidemic in the northwestern part of the ROK. There were clear differences in anti-Pv200 antibody responses between patient and non-patient groups. These results suggest that anti-Pv200 antibody detection by ELISA may be useful in diagnosing Korean $P$. vivax malaria in suspected patients. During the course of our study, we demonstrated the exis-

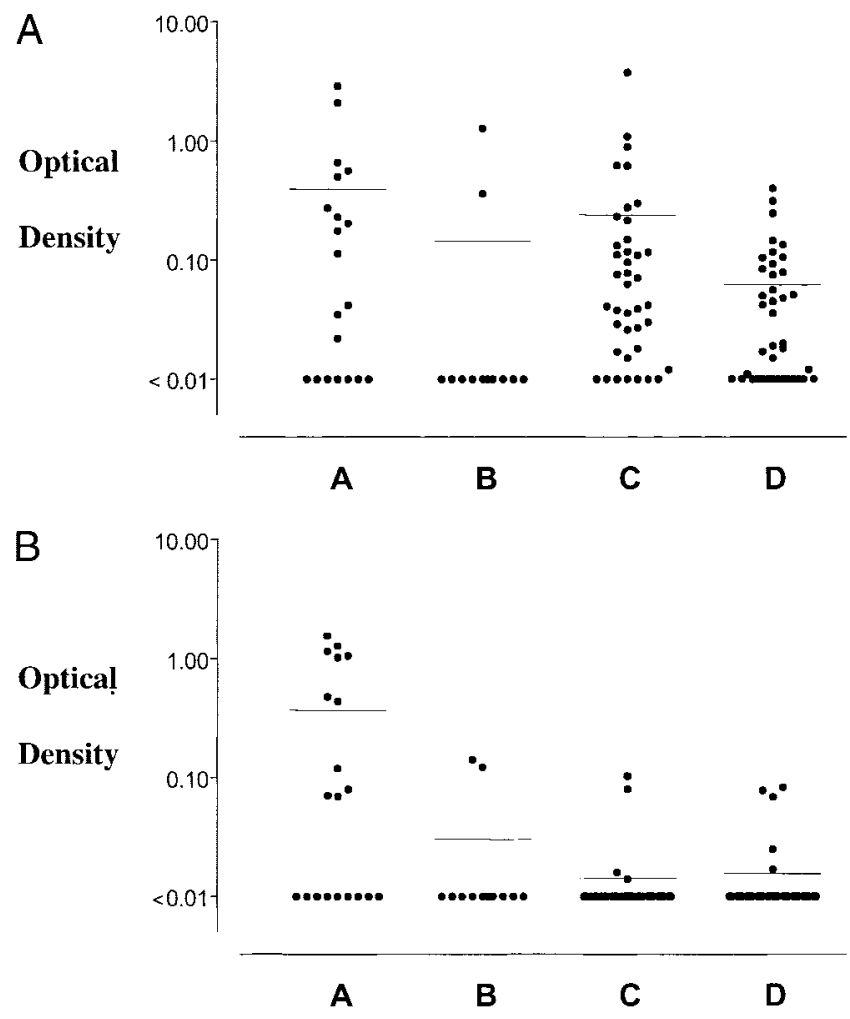

FIGURE 6. A): IgG isotype antibody levels against CS protein of Plasmodium vivax as determined by indirect enzyme-linked immunosorbent assay (ELISA) with V20 (CS capture antigen) in various groups. Group A: 20 malaria patients; B: 12 soldiers within 6 months after a malaria attack; C: 40 soldiers who had malaria attacks within 3 months following our sampling; D: 40 soldiers who did not have a malaria attack within 3 months following our sampling. The thin horizontal lines indicate the mean optical density (OD) values in anti-Pv200 IgG antibody titer. B): IgM isotype antibody levels against CS protein of Plasmodium vivax as determined by indirect ELISA with V20 (CS capture antigen) in the various groups as above. The thin horizontal lines indicate the mean OD values in anti-Pv200 IgM antibody titer.

\section{Amplified P.vivax ssrRNA gene}

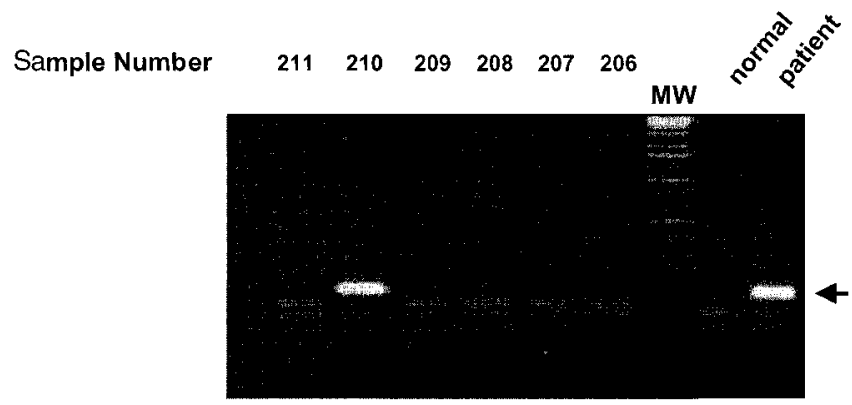

FIGURE 7. Ethidium bromide-stained $2 \%$ agarose gel showing an example of the amplified Plasmodium vivax ssrRNA gene fragment by nested polymerase chain reaction (PCR) from the whole blood samples of soldiers who had served in high-risk Plasmodium vivax malaria areas. A pool of 1,000 whole-blood samples were tested to detect the Plasmodium vivax specific ssrRNA gene with 4 samples identified as positive. Arrow indicates the positive result in samples; $\mathrm{MW}=$ DNA molecular weight marker; normal $=$ result of normal control whole blood; patient $=$ result of Plasmodium vivax patient whole blood prior to the drug treatment. 
tence of elevated levels of anti-Pv200 antibodies from several suspected patients who had not yet shown $P$. vivax on blood film smears, but who later showed positive results of $P$. vivax on blood film smears as malaria symptoms progressed. Further studies using larger sample sizes may be required to firmly establish the sensitivity and specificity of the method. One possible confusion in the interpretation of anti-Pv200 antibody titers is that some individuals may maintain high anti-Pv200 antibody titers for an extended period after recovery from malaria, and even after complete clearance of the parasite. According to the data from the prospective longitudinal study of 8 soldiers, most individuals with Korean $P$. vivax malaria maintain high anti-Pv200 antibody titers of the IgG isotype for 4 to 6 months after drug cure (Figure 3 ). It is possible that some individuals maintain high anti-Pv200 antibody titers for longer periods. The IgG isotype of anti-Pv200 antibodies had higher normal background levels (Figure 1) but a more consistent high response to infection (Figure 2) than did the IgM isotype. Although there is a danger of false negatives, the combination of both IgG and IgM isotype levels against Pv200 antigen could detect Korean patients infected with $P$. vivax, either in addition to, or as a replacement for traditional laboratory diagnosis.

One of the intriguing findings in the present study was that $27.5 \%$ of 40 soldiers who later developed symptomatic malaria had elevated anti-Pv200 antibody levels up to 3 months prior to illness (Figure 4). This implies that the antibody titers against Pv200, or MSP 1 antigen of $P$. vivax might have increased in the very early erythrocytic stage of infection, if not in the hepatic stage. All 40 soldiers were probably carrying $P$. vivax during a prolonged incubation period at the time of our sampling and could have been infected with $P$. vivax during the previous summer since all of the subjects had served in areas of high risk $P$. vivax transmission in the summer previous to our sampling, but were stationed in low-risk $P$. vivax areas thereafter. In support of this conclusion, the North Korean (NK) strain of $P$. vivax, characteristically, has a long incubation period in many patients. ${ }^{5}$ This phenomenon of a long dormancy has been widely and consistently observed in the course of the $P$. vivax malaria outbreak in the ROK. ${ }^{12}$ According to the epidemiologic survey conducted by the ROK Armed Forces Medical Command from 1996 through 1998 (Roh C-S and others, unpublished data), more than $60 \%$ of malaria patients in the ROK Armed Forces became infected with $P$. vivax during the summer of the previous year and became ill sometime after the winter and spring that followed. During these prolonged incubation periods of up to 1 year or longer, no apparent clinical signs and symptoms were observed. On the other hand, the rest of the malaria patients had short incubation periods typical of $P$. vivax malaria in many other parts of the world. Those malaria patients with a prolonged incubation period might play a very important role in maintaining infectious sources of $P$. vivax malaria for the following summer. Hence, the early detection of malaria patients in a prolonged incubation period could be crucial in trying to eradicate malaria from Korea. Anti-Pv200 antibody ELISA could be useful in the early detection of Korean $P$. vivax malaria in a certain portion of the population.

Anti-CS antibody levels in the various groups showed a broad range of values. Since the antibody titers in each group had diverse distributions, no clear cut-off values could be defined. CS protein can be classified into two groups according to the central 20 nonapeptide repeats: predominant type I repeats with a GDRADGQPA sequence and variant type II repeats with an ANGAGNQPG sequence. The use of different $P$. vivax CS proteins appears to be regionally biased. ${ }^{13,14}$ Therefore, the CS protein of $P$. vivax in the recent outbreak of malaria in the ROK is likely to have the sequence homologous to the NK strain (predominant type I repeats). In our results, however, antibody responses against CS protein with predominant type I repeats appeared to show no significant differences between patients and other groups (Figure 6). More than $50 \%$ of patients showed markedly lower anti-CS protein antibody titers (OD $<0.10)$. In addition to the poor antibody responses against CS protein of $P$. vivax after a prolonged incubation period, the possibility that those patients with low antibody titers against CS protein with type I repeats might have been infected with $P$. vivax strain carrying CS protein with variant type II repeats cannot be excluded.

The survey of 1,713 soldiers in high-risk malaria areas by anti-Pv200 antibody ELISA demonstrated that at least $10 \%$ of soldiers appeared to have been exposed to the malaria antigen. This ELISA test could be used in seroepidemiologic studies as an efficient tool for monitoring malaria prevalence and incidence in large cohorts.

As a result of the nested PCR of blood samples, we detected 4 soldiers $(0.4 \%)$ carrying $P$. vivax parasites who had no clinical signs and symptoms associated with $P$. vivax malaria at the time of our sampling. A few weeks later, 1 of those 4 soldiers was admitted to the field hospital for $P$. vivax malaria. The remaining 3 soldiers were also ordered to be treated with curative drugs for $P$. vivax malaria. Although anecdotal, these results indicate that asymptomatic blood carriers of $P$. vivax parasites are present in the Korean population.

The current epidemic of $P$. vivax malaria in Korea had an insidious beginning with only a single case detected in 1993. ${ }^{2}$ The medical community initially suggested that transmission in the ROK was due to mosquitoes flying across the border from the Democratic People's Republic of Korea (DPRK). ${ }^{3}$ However, although the majority of infection had occurred in soldiers stationed along the DPRK border, it was soon apparent that there was extensive transmission in the ROK civilian community. ${ }^{12,15}$ Without active surveillance, some civilian cases will inevitably go undetected because some people are either asymptomatic or suffer only mild symptoms. Though parasitemic, these infected people will not seek treatment and will therefore contribute to expansion of malaria parasite foci in Korea. Given the extremely long incubation period of Korean $P$. vivax in some individuals, ${ }^{1,12}$ infected people are more likely to travel throughout the peninsula before seeking treatment. Extensive geographic movement of the parasite has already occurred in American soldiers who carried the infection from Korea to the United States. ${ }^{1}$

Since the resurgence of epidemic malaria after a virtual absence of $P$. vivax transmission in the ROK for the last 20 years, we have a unique opportunity to study the serology of Korean $P$. vivax malaria in young subjects, most of whom have never experienced the threat of malaria before. To stop 
the current epidemic, a combination of vector control (Strickman D and others, unpublished data), personal protection, active case detection, and treatment are likely to be necessary. ${ }^{4}$ Considering the challenge of case detection caused by a long incubation period and the sometimes mild symptomatology of Korean $P$. vivax, the anti-Pv200 ELISA and nested PCR could be valuable tools in a program to systematically eradicate malaria from the Republic of Korea once again. These techniques could have been useful in assessing the extent of malarial resurgence earlier in the epidemic.

Acknowledgments: We thank Dr. Robert A. Wirtz for his generous support. We are enormously grateful to all the Republic of Korea soldiers who voluntarily participated in the study. We are also thankful to the medical and executive officers in the field, as well as in the ROK Armed Forces Medical Command, who helped us greatly in expediting the entire process. We thank the Korean Red Cross and the Korean Armed Forces Medical School for providing us with pools of normal serum samples. We are very grateful to the Department of Microbiology at Yonsei University College of Medicine for its generous support. We wish to express our utmost thanks to the soldiers, officers, and employees of the Korean Armed Forces Central Medical Research Institute.

Financial support: This work was supported in part by a KMMA (Korean Military Medical Association) grant for the fiscal year 1998.

Authors' addresses. Chae Gyu Park, Laboratory of Cellular Physiology and Immunology, Box 176, The Rockefeller University, 1230 York Avenue, New York, New York 10021; Yong-Joon Chwae, Department of Microbiology, College of Medicine, Pochon Cha University, 198-1 Dongkyo-ri, Pochon-eup, Pochon, Kyunggi, 487-800, Korea; Jong-Il Kim, Division of Gerontology, Department of Medicine, Beth Israel Deaconess Medical Center, Harvard Institutes of Medicine, Harvard Medical School, Boston, Massachusetts 02115; Ji-Ho Lee, Department of Occupational and Environmental Medicine, Ulsan University Hospital, 290-3 Cheonha-dong, Dong-gu, Ulsan, 682-060, Korea; Gang Min Hur, Department of Pharmacology, College of Medicine, Chungnam National University, 6 Munhwadong, Jung-gu, Taejon, 301-131, Korea; Byeong Hwa Jeon, Department of Physiology, College of Medicine, Chungnam National University, 6 Munhwa-dong, Jung-gu, Taejon, 301-131, Korea; Jae Soo Koh, Department of Anatomic Pathology, Korea Cancer Center Hospital, 215-4 Gongneung-dong, Nowon-gu, Seoul, 139-706, Korea; Jae-Hee Han, Department of Physiology, College of Medicine, Gyeongsang National University, 90 Chilam-dong, Chinju, Kyongnam, 660-751, Korea; Shin-Je Lee, Department of Microbiology, College of Medicine, Soonchunhyang University, 366-1 Ssang-yong Dong, Chunan, Chungnam, 330-090, Korea; Jae-Won Park, Korean Armed Forces Central Medical Research Institute, P. O. Box 78-503, Chumok-dong, Yuseong-gu, Taejeon, 305-153, Korea; David C. Kaslow, Merck and Co., Inc., Virus and Cell Biology, WP26A, Rm 4044, MSC-4000, Merck Research Labs, West Point, Pennsylvania 19486-0004; Daniel Strickman, Department of Entomology, Walter Reed Army Institute of Research, Bldg. 503, Robert Grant Avenue, Silver Spring, Maryland 20910; and Cheon-Seop Roh, Bureau of Medical Affairs, The First Field Army Command of the Republic of Korea Army, Wonju, Kangwon, 220-799, Korea.
Reprint requests: Chae Gyu Park, PhD, Laboratory of Cellular Physiology and Immunology, Box 176, The Rockefeller University, 1230 York Avenue, New York, NY 10021; Tel: 212-327-8114; Fax: 212 327-8875; E-mail: parkc@mail.rockefeller.edu.

\section{REFERENCES}

1. Feighner BH, Park SI, Novakoski WL, Kelsey LL, Strickman D, 1998. Reemergence of Plasmodium vivax malaria in the Republic of Korea. Emerg Infect Dis 4: 295-297.

2. Chai IH, Lim GI, Yoon SN, Oh WI, Kim SJ, Chai JI, 1994 Occurrence of tertian malaria in a male patient who has never been abroad. Korean J Parasitol 32: 195-200.

3. Cho SY, Kong Y, Park SM, Lee JS, Lim YA, Chae SL, Kho WG, Lee JS, Shim JC, Shin HK, 1994. Two vivax malaria cases detected in Korea. Korean J Parasitol 32: 281-284.

4. Paik YH, Ree HI, Shim JC, 1988. Malaria in Korea. Japan J Exp Med 58: 55-56.

5. Shute PG, Lupascu G, Branzei P, Maryon M, Constantinescu P, Bruce-Chwatt LJ, Draper CC, Killick-Kendrick R, Garnham PC, 1977. A strain of Plasmodium vivax characterized by prolonged incubation: the effect of numbers of sporozoites on the length of the prepatent period. Trans $R$ Soc Trop Med Hyg 70: 474-481.

6. Wirtz RA, Duncan JF, Njelesani EK, Schneider I, Brown AE, Oster CN, Were JBO, Webster HK, 1989. ELISA method for detecting Plasmodium falciparum circumsporozoite antibody. Bull World Health Organ 67: 535-542.

7. Carlos A, Espinal CA, Morales PO, 1984. Indirect ELISA test for malaria in blood donors. Trans $R$ Soc Trop Med Hyg 78: 645-647.

8. Gonzalez-Ceron L, Rodriguez MH, 1991. An enzyme-linked immunosorbent assay using detergent-soluble Plasmodium vivax antigen for seroepidemiological surveys. Trans $R$ Soc Trop Med Hyg 85: 358-361.

9. Kaslow DC, Kumar S, 1996. Expression and immunogenicity of the C-terminal of a major blood-stage surface protein of Plasmodium vivax, Pv200 19 , secreted from Saccharomyces cerevisiae. Immunol Lett 51: 187-189.

10. Wirtz RA, Rosenberg R, Sattabongkot J, Webster HK, 1990. Prevalence of antibody to heterologous circumsporozoite protein of Plasmodium vivax in Thailand. Lancet 336: 593-595.

11. Snounou G, Viriyakosol S, Zhu XP, Jarra W, Pnheiro L, Rosario VED, Thaithong S, Brown NK, 1993. High sensitivity of detection of human malaria parasites by the use of nested polymerase chain reaction. Mol Biochem Parasitol 61: 315-320.

12. Lee JS, Kho WG, Lee HW, Seo M, Lee WJ, 1998. Current status of vivax malaria among civilians in Korea. Korean J Parasitol 36: $241-248$.

13. Qari SH, Goldman IF, Povoa MM, Oliveira S, Alpers MP, Lal AA, 1991. Wide distribution of the variant form of the human malaria parasite Plasmodium vivax. J Biol Chem 266: 1629716300.

14. Mann VH, Huang T, Cheng Q, Saul A, 1994. Sequence variation in the circumsporozoite protein gene of Plasmodium vivax appears to be regionally biased. Mol Biochem Parasitol 68: 45-52.

15. Strickman D, Miller ME, Kelsey LL, Lee WI, Lee HW, Lee KW, Kim HC, Feighner BH, 1999. Evaluation of the malaria threat at the Multi-Purpose Range Complex, Yongpyong, Republic of Korea. Military Med 164: 626-629. 